\title{
COMPARAÇÃO DE TRÊS EQUAÇÕES PARA PREDIÇÃO DA GORDURA CORPORAL POR BIOIMPEDÂNCIA EM JOVENS OBESAS
}

\author{
COMPARISON OF THREE EQUATIONS FOR PREDICTING THE BODY FAT BY BIOIMPEDANCE \\ IN YOUNG OBESE FEMALES
}

\section{COMPARACIÓN DE TRES ECUACIONES PARA PREDICCIÓN DE LA GRASA CORPORAL POR BIOIMPEDANCIA EN JÓVENES OBESAS}

\author{
Wendell Arthur Lopes \\ (Educador Físico) \\ Neiva Leite (Médica) \\ Larissa Rosa da Silva² \\ (Educadora Física) \\ Cássio Leandro Mühe Consentino ${ }^{2}$ \\ (Educador Físico) \\ Priscilla Coutinho 3 (Médica) \\ Rosana Bento Radominski³ (Médica) \\ Cláudia Regina Cavaglieri ${ }^{4}$ \\ (Bioquímica) \\ 1. Universidade Estadual de \\ Maringá, Departamento de \\ Educação Física, Maringá, PR, Brasil. \\ 2. Universidade Federal do Paraná \\ (UFPR), Departamento de Educação \\ Física, Curitiba, PR, Brasil. \\ 3. Universidade Federal do Paraná \\ (UFPR), Departamento de Nutrição, \\ Curitiba, PR, Brasil. \\ 4. Universidade Estadual de \\ Campinas, Faculdade de Educação \\ Física, Campinas, São Paulo, Brasil.
}

\section{Correspondência:}

Rua Domingos Caetano do Amaral, 2417, Bairro Batel, Guarapuava, PR, Brasil. 85015-354.

warthurlopes@gmail.br

\section{RESUMO}

Introdução: A impedância bioelétrica (BIA) tem sido bastante utilizada para o monitoramento da composição corporal em indivíduos de diferentes idades e estados nutricionais. Entretanto, não se sabe qual das equações propostas para crianças e adolescentes é a mais recomendada para utilização em adolescentes com excesso de peso. Objetivo: Verificar a concordância dos métodos de BIA usando três equações diferentes com a absorciometria de raio-X de dupla energia (DXA), para análise da composição corporal de adolescentes com sobrepeso e obesidade. Métodos: Participaram do estudo 27 adolescentes do sexo feminino, com sobrepeso e obesidade. Foram avaliados o percentual de gordura corporal (\%GC), a massa gorda (MG) e a massa livre de gordura (MLG) por DXA e por BIA utilizando as equações propostas por Houtkooper, Schaefer e Deurenberg. ANOVA oneway, gráficos de Bland-Altman e o coeficiente de correlação intraclasse foram utilizados para comparação e verificação da concordância entre os métodos. Resultados: A BIA utilizando a equação proposta por Houtkooper foi a única que não apresentou diferença estatística significativa na estimativa de \%GC, MG e MLG em comparação ao DXA e apresentou boa concordância com o DXA na estimativa de \%GC $(-1,9 \pm 3,29 \%), M G(1,5 \pm 2,59 \mathrm{~kg})$ e MLG $(1,4 \pm 2,60 \mathrm{~kg})$, bem como boa reprodutibilidade para \%GC (CCl = $0,81), M G(0,96)$ e MLG $(0,89)$. As equações de Schaefer e de Deurenberg apresentaram menor concordância com o DXA, superestimando a MG e subestimando a MLG e apresentaram reprodutibilidade de moderada a baixa na maioria das medidas da composição corporal. Conclusão: Quando comparamos as três equações propostas para BIA com o DXA, verificamos que a equação proposta por Houtkooper foi a que melhor concordou com DXA e apresentou boa reprodutibilidade para estimar \%GC, MG e MLG em adolescentes com sobrepeso e obesidade.

Palavras-chave: impedância elétrica, obesidade, adolescente, composição corporal.

\section{ABSTRACT}

Introduction: The bioelectrical impedance (BIA) has been widely used for monitoring body composition of individuals of different ages and nutritional statuses. However, it is unknown which of the equations for children and adolescents is the most recommended to be used for adolescents who are overweight. Objective: To assess the agreement of the BIA method using three different equations with dual energy X-ray absorptiometry (DXA) for analysis of body composition in overweight and obesity. Methods: Twenty-seven female adolescents with overweight or obesity were included in this study. The percentage of body fat (\%BF), fat mass (FM) and fat-free mass (FFM) were evaluated by DXA and BIA using the equations proposed by Houtkooper, Schaefer and Deurenberg. Oneway ANOVA, Bland-Altman plots and the intraclass correlation coefficient were used for comparison and verification of the agreement among the methods. Results: BIA using the equation proposed by Houtkooper was the only one that showed no statistically significant difference in the estimating \%BF, FM and FFM compared to DXA and showed good

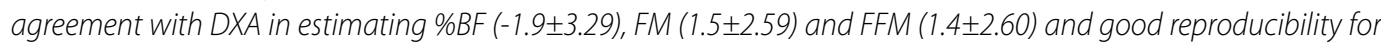
$\% B F(0.81), F M(0.96)$ and FFM (0.89). The Schaefer and Deurenberg equations showed less agreement with DXA, overestimating the $M G$ and underestimating the FFM, and presented moderate to low reproducibility in most measures of body composition. Conclusion: When we compared the three equations proposed to BIA with DXA, we find that the Houtkooper equation was the best agreed with DXA and showed good reproducibility for estimate \%BF, FM and FFM in female adolescents with overweight and obesity.

Keywords: electric impedance, obesity, adolescents, body composition.

\section{RESUMEN}

Introducción: La impedancia bioeléctrica (BIA) ha sido bastante utilizada para el monitoreo de la composición corporal en individuos de diferentes edades y estado nutricional. Entretanto, no se sabe cuál de las ecuaciones propuestas para niños y adolescentes es la más recomendada para uso en adolescentes con exceso de peso. Objetivo: 
Verificar la concordancia de los métodos de BIA usando tres ecuaciones diferentes con la absormetría de rayos X de doble energía (DXA), para análisis de la composición corporal de adolescentes con sobrepeso y obesidad. Métodos: Participaron en el estudio 27 adolescentes, del sexo femenino, con sobrepeso y obesidad. Fueron evaluados el porcentual de grasa corporal (\%GC), la masa gorda (MG) y la masa libre de grasa (MLG) por DXA y por BIA utilizando las ecuaciones propuestas por Houtkooper, Schaefer y Deurenberg. ANOVA oneway, Bland-Altman y el coeficiente de correlación intraclase fueron utilizados para comparación y verificación de la concordancia entre los métodos. Resultados: La BIA utilizando la ecuación propuesta por Houtkooper fue la única que no presentó diferencia estadística significativa en la estimativa de \%GC, MG y MLG en comparación a DXA y presentó buena concordancia con

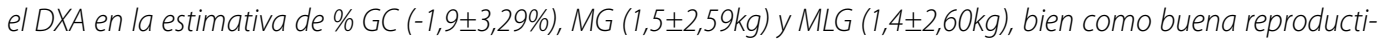
bilidad para \%GC (CCl=0,81), MG (0,96) y MLG (0,89). Las ecuaciones Schaefer y de Deurenberg presentaron menor concordancia con el DXA, sobreestimando la MG y subestimando la MLG y presentaron valores de moderada a baja reproductibilidad en la mayoría de las medidas de la composición corporal. Conclusión: Cuando comparamos las tres ecuaciones propuestas para BIA en comparación al DXA, verificamos que la ecuación propuesta por Houtkooper fue la que mejor concordó con DXA y presentó buena reproductibilidad para estimar \%GC, MG y MLG en adolescentes con sobrepeso y obesidad.

Palabras clave: impedancia eléctrica, obesidad, adolescente, composición corporal.

\section{INTRODUÇÃO}

O sobrepeso e obesidade na adolescência têm preocupado os profissionais da área da saúde devido ao aumento da sua prevalência nas últimas décadas ${ }^{1}$ e a associação com outras doenças crônicas não transmissíveis (DCNTs) como diabetes, hipertensão arterial, dislipidemia e disfunção endotelial ${ }^{2}$. O tratamento da obesidade nessa fase é difícil e as estratégias mais eficazes envolvem mudanças no estilo de vida, principalmente a aquisição de melhores hábitos alimentares e a prática de exercícios físicos ${ }^{3}$. Estas ações podem induzir a melhora na composição corporal, mas pouca ou nenhuma redução de peso corporal $^{4}$. Portanto, o uso de ferramentas adequadas para estimativa da composição corporal torna-se imprescindível para verificar os efeitos de um programa de intervenção nessa população.

A densitometria de duplo feixe de raios $X$ (DXA) é uma técnica avançada para estimar a gordura corporal, massa livre de gordura, bem como o conteúdo e densidade mineral óssea, sendo o principal método indicado para população pediátrica, devido à mínima exposição à radiação e praticidade de aplicação ${ }^{5}$. No entanto, a utilidade do DXA é limitada devido ao custo do equipamento, necessidade de pessoal especializado para operá-lo, a falta de portabilidade e suas limitações quanto ao tamanho corporal do avaliado (peso máximo suportado de 120 kg), o que dificulta a avaliação da composição corporal de obesos.

A análise de impedância bioelétrica (BIA) se apresenta como uma alternativa mais aplicável para avaliação da composição corporal na população geral. Em comparação ao DXA, este método demonstra algumas vantagens, como ser relativamente barato, portátil, requer uma formação mínima de operar, e não apresenta qualquer risco para a saúde dos participantes. Dessa maneira, a BIA tem sido amplamente utilizada pelos profissionais da saúde e várias equações estão disponíveis para estimativa da composição corporal de crianças e adolescentes ${ }^{6}$ e adultos. Entretanto, estudos tem mostrado que a utilização dessas equações na população obesa tem subestimado a gordura corporal e superestimado a massa livre de gordura ${ }^{7}$. Além disso, poucos estudos têm desenvolvido e validado equações específicas para obesos ${ }^{8}$ e a sua utilização é limitada devido à faixa etária e ao grau de obesidade a qual a equação foi derivada. A investigação da validade preditiva das equações desenvolvidas para crianças e adolescentes pode oferecer informações importantes e permitir aos profissionais a escolha de equações adequadas para estimativa da composição corporal de crianças e adolescentes com sobrepeso e obesidade.

Nessa perspectiva, Cleary et al. ${ }^{9}$ verificaram a validade preditiva de quatro equações desenvolvidas para utilização em crianças com sobrepeso e obesidade $(7,6 \pm 1,28$ anos) e encontraram que somente a equação de Schaefer et al. ${ }^{10}$ foi acurada para estimativa da gordura corporal dessa população. Até o momento, nenhum estudo verificou a validade preditiva dessas equações em adolescentes com sobrepeso e obesidade. Portanto, o objetivo do presente estudo foi avaliar a validade preditiva de três equações para estimativa da gordura corporal desenvolvidas para crianças e adolescentes em uma amostra de adolescentes com sobrepeso e obesidade.

\section{MÉTODOS}

Trata-se de um estudo transversal composto por 27 adolescentes do sexo feminino com excesso de peso, com média de idade de 14,4 1,11 anos, selecionados por conveniência e provenientes de escola pública de Curitiba/Paraná. Os critérios de inclusão do estudo foram: sexo feminino, idade entre 13 e 17 anos, estágio de Tanner entre IV e $\mathrm{V}$ e presença de excesso de peso (IMC escore-z $>+1$ ). Os participantes não apresentavam nenhuma contraindicação para realização dos exames, incluindo ausência de doenças cardíacas, pulmonares e metabólicas. Os voluntários não realizavam outra atividade física regular, nos últimos seis meses, além da educação física escolar, bem como não participavam de nenhum tratamento para redução de peso nos últimos três meses e não tinham histórico de perda de peso recente. Todos os voluntários e os pais ou responsáveis foram informados e assinaram o Termo de Consentimento Livre e Esclarecido, conforme projeto de pesquisa aprovado pelo Comitê de Ética em Pesquisa em Seres Humanos (protocolo n 2460.067/2011-03).

A massa corporal ( $\mathrm{kg}$ ) foi aferida em balança Filizola ${ }^{\circledR}$ (Brasil), com precisão de 0,1 kg, tipo plataforma, com o avaliado permanecendo em posição ortostática, descalço, pés unidos no centro da plataforma e vestindo roupas leves. A estatura $(\mathrm{cm})$ foi aferida em estadiômetro fixado à balança, com precisão de 0,1cm, estando o indivíduo descalço, pés unidos no centro da plataforma, cabeça posicionada no plano horizontal de Frankfort ao final de inspiração máxima. $O$ índice de massa corporal (IMC) foi calculado pela divisão da massa corporal (MC) pela estatura ao quadrado (IMC=MC/estatura ${ }^{2}$ ). A classificação do excesso de peso foi realizada com base no cálculo do IMC escore- $Z$, sendo considerado excesso de peso os valores acima do IMC escore- $Z+1$, conforme proposta da Organização Mundial da Saúde ${ }^{11}$.

A avaliação puberal foi realizada por inspeção visual, realizada por médico treinado com base no estadiamento proposto por Tanner ${ }^{12}$, classificando 
o desenvolvimento mamário (M1-M5) e/ou pilificação pubiana (P1-P5).

Para avaliação da composição corporal foram utilizados os métodos de DXA e a BIA, determinando o percentual de gordura (\%GC), a gordura corporal (GC) e a massa livre de gordura (MLG).

Para avaliação da composição corporal por DXA foi utilizado o aparelho da marca Lunar ${ }^{\circledR}$, modelo Prodigy Primo (General Electric Healthcare; Madison, WI). O exame foi realizado com o indivíduo deitado em decúbito dorsal sobre uma mesa, onde a fonte e o detector foram passados através do corpo com uma velocidade relativamente lenta de $1 \mathrm{~cm} / \mathrm{s}$. A duração do escaneamento foi de aproximadamente 5 minutos e o tempo total do exame de 15 minutos, considerando as instruções iniciais, o escaneamento do corpo todo e a emissão do resultado. Os sujeitos foram orientados a estarem vestidos com roupas livres de objetos metálicos, bem como retirarem assessórios de metal presente no corpo (brincos, piercing, anel, entre outros) que poderiam interferir no exame. Para a reconstrução da imagem dos tecidos subjacentes e quantificação de conteúdo mineral ósseo, da massa de gordura total e da massa corporal isenta de gordura foi utilizado o software enCORE 2008 (General Electric Healthcare; Madison, WI).

Para o método de impedância bioelétrica (BIA) foi utilizado o aparelho Maltron ${ }^{\circledR}$, modelo BF906 (Maltron; Essex, UK), tetrapolar, com frequência de $50 \mathrm{khz}$. O procedimento foi realizado no período da manhã, após jejum de 10-12 horas, em decúbito dorsal. Os eletrodos foram posicionados nas superfícies dorsais das mãos e dos pés direitos, próximo às articulações metacarpo-falangeanas e metatarso-falangeanas e medialmente entre as proeminências distais do rádio e da ulna, entre o maléolo tibial e fibular. Obteve-se a resistência e calculou-se a massa livre de gordura (MLG) por meio das equações propostas por Houtkooper et al. ${ }^{13}\left[\mathrm{MLG}=0,61 \times\left(\mathrm{E}^{2} / \Omega\right)+(0,25 \times \mathrm{MC})+1,31\right]$, Schaefer et $a l^{10}\left[\mathrm{MLG}=0,65 \times\left(E^{2} / \Omega\right)+(0,68 x \mathrm{l})+0,15\right]$ e Deurenberg et al. ${ }^{14}$ $\left[\mathrm{MLG}=0,64 \times\left(\mathrm{E}^{2} / \Omega\right)+4,83\right]$, onde $\mathrm{E}=$ estatura $(\mathrm{cm}), \mathrm{MC}=$ massa corporal, I=idade (anos) e $\Omega=$ resistência.

\section{Análise Estatística}

Para análise dos dados foram utilizadas a ANOVA oneway seguido do teste de comparações múltiplas de Bonferroni, o método de Bland-Altman e o cálculo do coeficiente de correlação intraclasse (CCI). 0 pacote estatístico foi SPSS versão 13.0 e Prisma versão 5.0. O nível de significância adotado foi $p<0,05$.

\section{RESULTADOS}

Participaram do estudo 27 adolescentes. Todas as participantes apresentaram estágio maturacional entre 4 e 5 (6/21). A média de idade foi 14,8 $\pm 1,1$ anos e as médias de peso e estatura foram $74,1 \pm 11,3 \mathrm{~kg}$ e $1,61 \pm 0,06 \mathrm{~m}$, respectivamente. A média do IMC foi $28,5 \pm 3,2 \mathrm{~kg} / \mathrm{m}^{2}$. A frequência de sobrepeso e obesidade foi de 13/14, respectivamente.

Na tabela 1, encontram-se as comparações entre os valores do percentual de gordura corporal (\%GC), massa gorda (MG) e massa livre de gordura (MLG) estimada pelas três equações utilizadas na BIA e pelo DEXA. Com exceção da equação Houtkooper et al. ${ }^{13}$ (\%GC $t=0,81$, $p>0,05 ; M G t=1,42, p>0,05 ; M L G t=0,59, p>0,05)$, as equações Schaefer et al. ${ }^{10}$ e Deurenberg et al. ${ }^{14}$ diferiram significativamente dos valores obtidos pelo DEXA (tabela 1).

Na figura 1, encontra-se a representação gráfica pelo método de Bland-Altman da concordância e limites de concordância na estimativa do percentual da gordura corporal (\%GC), entre o DEXA e as equações de Houtkooper et al. ${ }^{13}$, Schaefer et al. ${ }^{10}$ e de Deurenberg et al..$^{14}$ para a BIA. A equação de Houtkooper et al. ${ }^{13}$ subestimou o \%GC em 1,9\%. Por outro lado, as equações de Schaefer et al. ${ }^{10}$ e de Deurenberg et al. ${ }^{14}$ superestimaram o \%GC em 8,8 e 16,7\%, respectivamente.

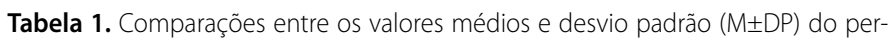
centual de gordura corporal, gordura corporal e massa livre de gordura estimada pelas três equações utilizadas na BIA e pelo DXA obtidos em adolescentes com excesso de peso $(n=27)$.

\begin{tabular}{|c|c|c|c|c|c|c|}
\hline & $\begin{array}{c}\text { DEXA } \\
(\mathrm{M} \pm \mathrm{DP})\end{array}$ & $\begin{array}{c}\text { Houtkooper }^{13} \\
(\mathrm{M} \pm \mathrm{DP})\end{array}$ & $\begin{array}{c}\text { Schaefer }^{10} \\
(M \pm D P)\end{array}$ & $\begin{array}{c}\text { Deurenberg }^{14} \\
(\mathrm{M} \pm \mathrm{DP})\end{array}$ & $F$ & $P$ \\
\hline$\% \mathrm{GC}$ & $43,8 \pm 4,9$ & $41,9 \pm 4,3$ & $52,7 \pm 5,9^{* * *}$ & $60,5 \pm 5,1^{* * *}$ & 54,08 & $<0,0001$ \\
\hline $\mathrm{MG}(\mathrm{kg})$ & $32,9 \pm 7,9$ & $31,4 \pm 7,5$ & $39,6 \pm 9,9^{*}$ & $45,3 \pm 10,2^{* * *}$ & 13,80 & $<0,0001$ \\
\hline $\mathrm{MLG}(\mathrm{kg})$ & $41,3 \pm 4,6$ & $42,7 \pm 4,6$ & $34,6 \pm 2,7^{* * *}$ & $28,8 \pm 2,4^{* * *}$ & 79,23 & $<0,0001$ \\
\hline
\end{tabular}

$\mathrm{DEXA}=$ absormetria de raio-X de dupla energia;B|A=impedância bioelétrica; $M G=$ massa gorda; $M L G=$ massa livre de gordura; "diferença significativa em relação ao DEXA;"0,05, "“"0,001.

A

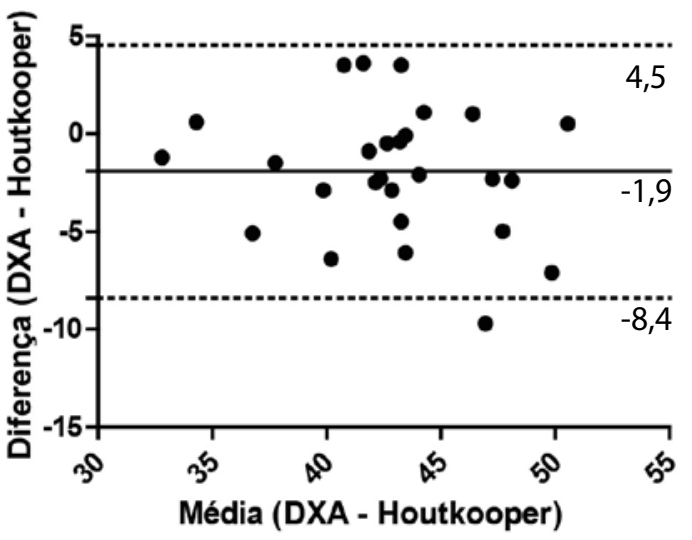

B

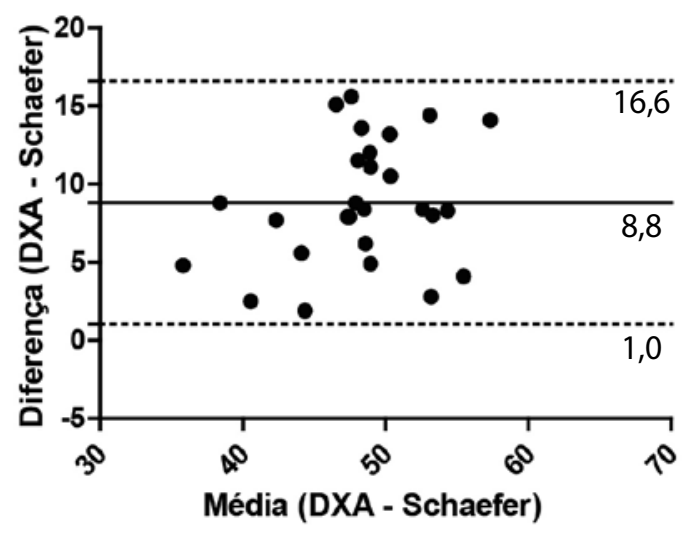

C

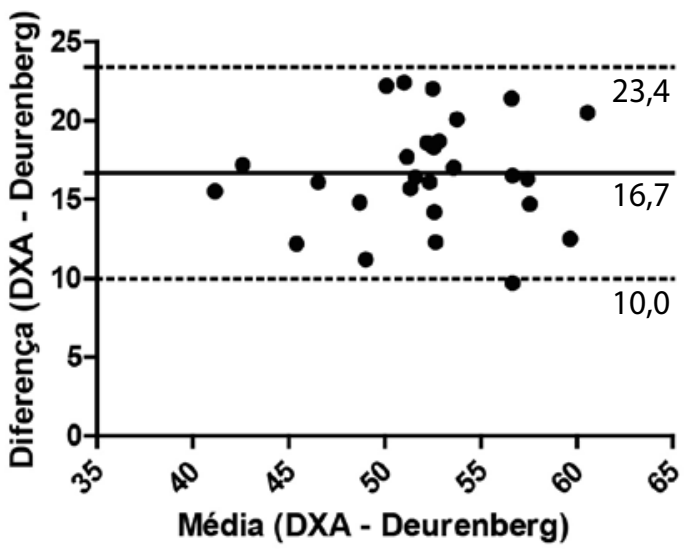

Figura 1. Bland-Altman mostrando a concordância (linha continuada) e limites de concordância (linha pontilhada) entre o DEXA e as equações de Houtkooper et al. ${ }^{13}$ (A), Schaefer et al..$^{10}$ (B) e Deurenberg et al. ${ }^{14}$ (C) utilizadas pela BIA para a estimativa do percentual de gordura corporal (\%GC) em adolescentes com excesso de peso. 
Na figura 2, encontra-se a representação gráfica pelo método de Bland-Altman da concordância e limites de concordância na estimativa da massa gorda (MG), entre o DEXA e as equações de Houtkooper et al. ${ }^{13}$, Schaefer et al. ${ }^{10}$ e de Deurenberg et al. ${ }^{14}$ para BIA. A equação de Houtkooper et al. ${ }^{13}$ subestimou a MG em 1,5 kg, ao passo que as equações de Schaefer et al. ${ }^{10}$ e de Deurenberg et al. ${ }^{14}$ superestimaram a MG em 6,7 e 12,4 kg, respectivamente.

Na figura 3, encontra-se a representação gráfica pelo método de Bland-Altman da concordância e limites de concordância na estimativa da massa livre de gordura (MLG), entre o DEXA e as equações de Houtkooper et al. ${ }^{13}$, Schaefer et al. ${ }^{10}$ e de Deurenberg et al. ${ }^{14}$

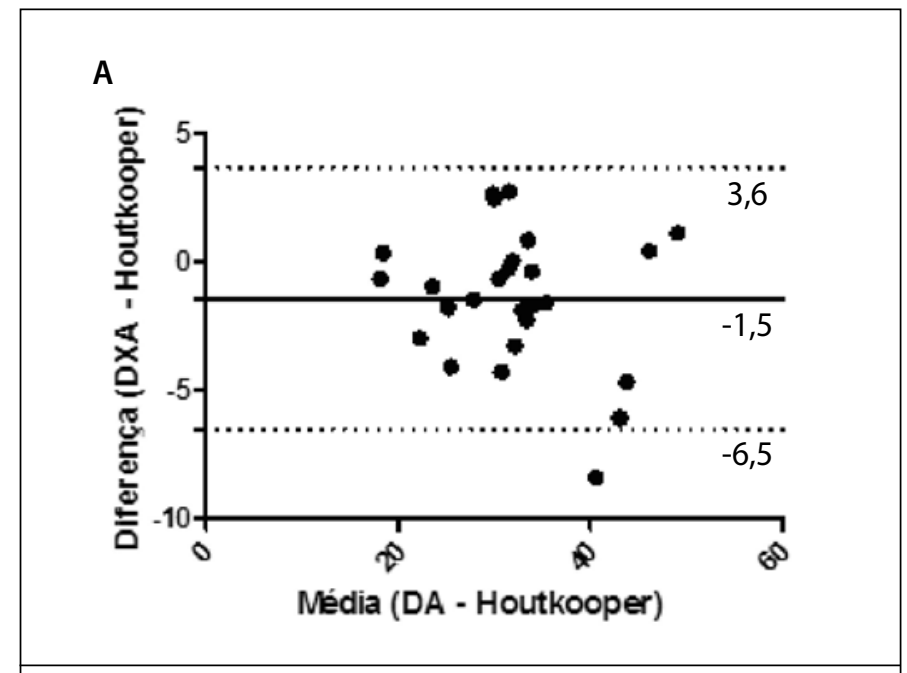

\section{B}

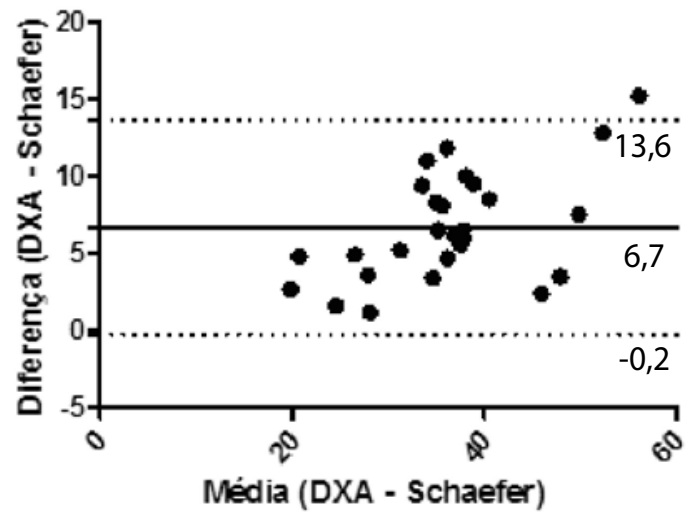

C

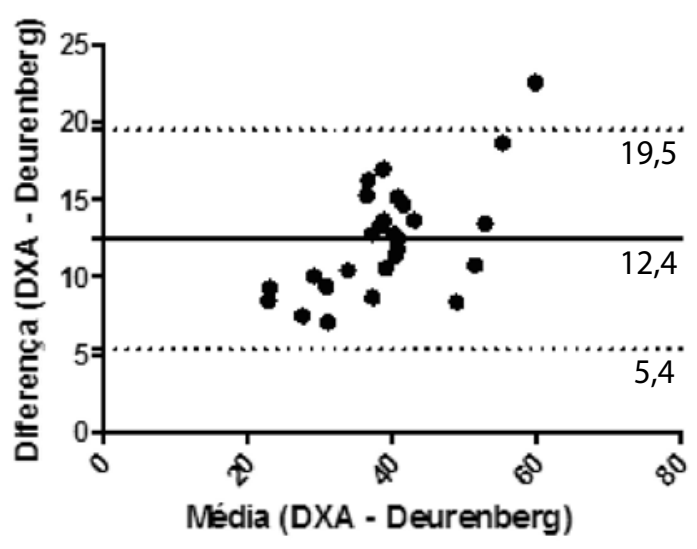

Figura 2. Bland-Altman mostrando a concordância (linha continuada) e limites de concordância (linha pontilhada) entre o DEXA e as equações de Houtkooper et al. ${ }^{13}$ (A), Schaefer et al..$^{10}$ (B) e Deurenberg et al..$^{14}$ (C) utilizadas pela BIA para a estimativa da massa gorda (MG) em adolescentes com excesso de peso. para BIA. A equação Houtkooper et al. ${ }^{13}$ superestimou a MLG em $1,4 \mathrm{~kg}$ quando comparado ao DEXA. Por outro lado, as equações Schaefer et al. ${ }^{10}$ e Deurenberg et al. ${ }^{14}$ subestimaram a MLG em 6,7 e 12,4 kg, respectivamente.

Os resultados do $\mathrm{CCl}$ indicaram boa reprodutibilidade entre a equação de Houtkooper et al. ${ }^{13}$ e o DEXA para \%GC $(0,81)$, MG $(0,96)$ e MLG $(0,89)$. A equação de Schaefer et al. ${ }^{10}$ apresentou boa reprodutibilidade apenas para a MG $(0,84)$, moderada para o \%GC $(0,48)$ e baixa para a MLG $(0,37)$. A equação de Deurenberg et al. ${ }^{14}$ apresentou moderada reprodutibilidade para MG $(0,65)$ e baixa reprodutibilidade para o \%GC $(0,21)$ e para a MLG $(0,15)$.

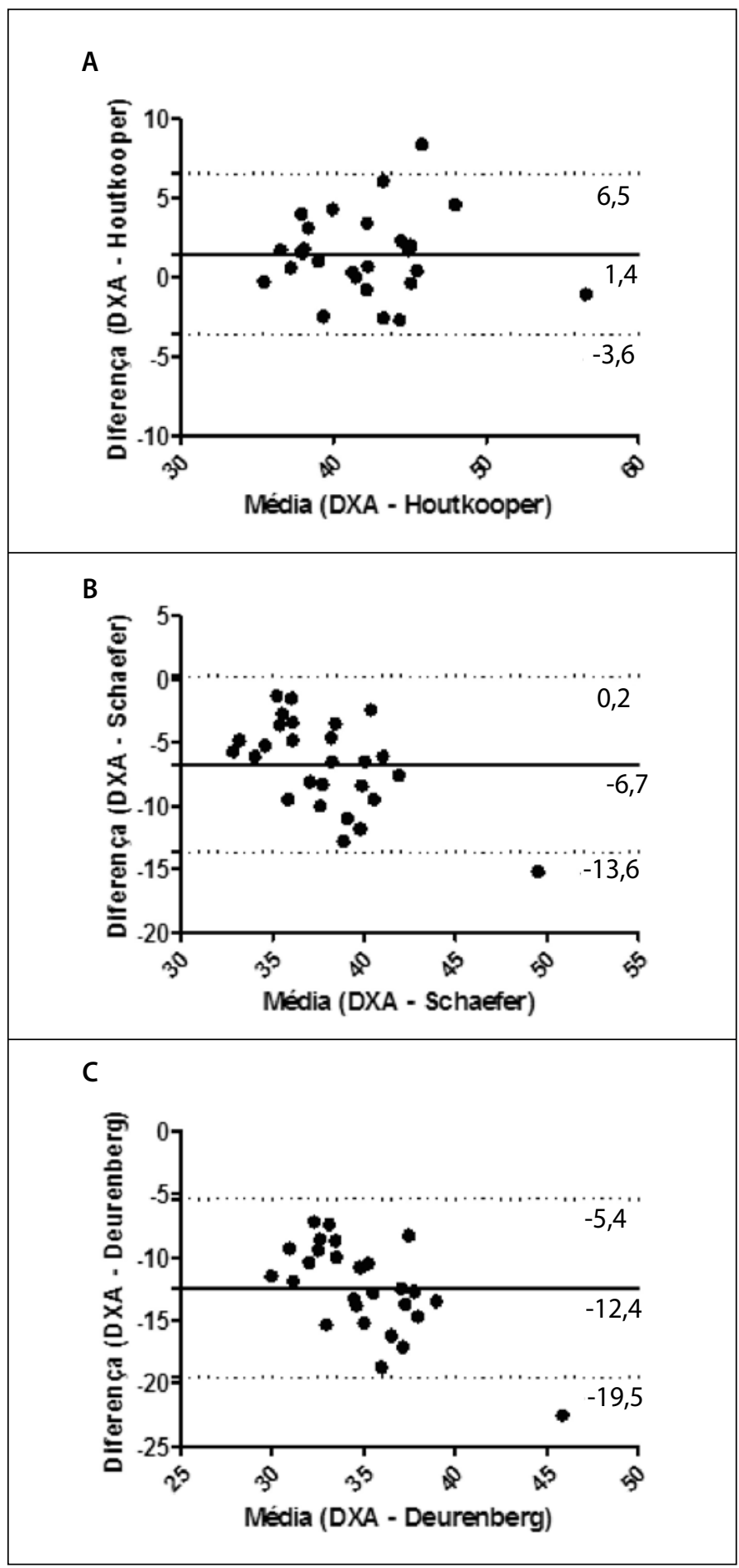

Figura 3. Bland-Altman mostrando a concordância (linha continuada) e limites de concordância (linha pontilhada) entre o DEXA e as equações de Houtkooper et al. ${ }^{13}$ (A), Schaefer et al. ${ }^{10}$ (B) e Deurenberg et al..$^{14}$ (C) utilizadas pela BIA para a estimativa da massa livre de gordura (MLG) em adolescentes com excesso de peso. 


\section{DISCUSSÃO}

Dentre as equações utilizadas neste estudo, apenas a equação BIA-Houtkooper ${ }^{13}$ apresentou boa concordância e reprodutibilidade com a absormetria de raio-X de dupla energia (DEXA) para estimativa do percentual de gordura (\%GC), da massa gorda (MG)e da massa livre de gordura (MLG) em adolescentes com sobrepeso e obesidade.

Estudos que investigaram a estimativa da composição corporal pela BIA em comparação ao DEXA em indivíduos obesos tem encontrado que este método subestima a MG e superestima a $\mathrm{MLG}^{6-7}$. No presente estudo, a equação proposta por Houtkooper et al. ${ }^{13}$ também subestimou a MG (-1,5 kg) e superestimou a MLG (+1,4 kg), corrroborando com esses estudos. As explicações para essas diferenças são a tendência em superestimar a água da MLG e a distribuição peculiar da MG no corpo de indivíduos obesos 9 . A suposição de que 73,2\% da MLG é composta de água corporal total não é totalmente correta. Em pessoas saudáveis, o teor de água na MLG é constante apenas após a idade de 20 anos. Na obesidade, no entanto, a hidratação da MLG pode ser de até 75\%. Este fato poderia causar uma superestimação da MLG, que por sua vez poderia subestimar o MG quando utilizada a técnica da BIA.

No presente estudo, a equação BIA-Houtkooper ${ }^{13}$ superestimou a MLG. Por outro lado, as equações BIA-Schaefer e BIA-Deurenberg subestimaram a MLG em 6,7 e 12,4kg, respectivamente. A discrepância na estimativa da composição corporal entre as equações utilizadas no presente estudo podem estar relacionadas a ampla variação na população da qual essas equações foram validadas. A equação proposta por Duremberg et al. ${ }^{14}$ foi desenvolvida a partir de uma amostra com idade entre 7 e 83 anos de idade. Schaefer et al. ${ }^{10}$ utilizou apenas crianças e adolescentes, porém, também utilizando uma ampla faixa de idade (3,9 a 19,3 anos). A equação de Houtkooper et al. ${ }^{13}$ desenvolveu a equação utilizando população jovens, numa faixa mais estreita de idade (10 a 19 anos).

No estudo de Cleary et al. ${ }^{9}$ a equação de Schaefer et al. ${ }^{10}$ foi a mais acurada para estimativa da gordura corporal de crianças com sobrepeso e obesidade. Porém, no presente estudo a equação de Schaefer et al. ${ }^{10}$ superestimou o percentual de gordura em $8,8 \%$ e subestimou a massa livre de gordura em 6,7 kg. Portanto, apesar da equação de Schaefer et al. ${ }^{10}$ ter sido desenvolvida para aplicação em crianças e adolescentes, esta equação é acurada para estimativa da composição corporal em crianças obesas, mas não em adolescentes obesos como evidenciado neste estudo. No presente estudo, apenas a equação de Houtkooper et al. ${ }^{13}$ não diferiu dos valores estimados pelo DEXA e apresentou meIhores valores de concordância e de reprodutibilidade que as outras equações utilizadas.

A novidade deste estudo foi que o uso da BIA utilizando a equação de Houtkooper et al..$^{13}$ permite uma estimativa da composição corporal com boa concordância e reprodutibilidade em adolescentes com sobrepeso e obesidade. Entretanto, uma das limitações do presente estudo foi a utilização de adolescentes somente do sexo feminino, o que não permite a generalização desses resultados ao sexo masculino. Esses achados tem implicações práticas importantes, considerando o baixo custo e facilidade na utilização da BIA para avaliação e controle da composição corporal de adolescentes devido ao aumento da obesidade juvenil. Além disso, devido a inexistência de equações exclusivas para adolescentes brasileiros com excesso de peso, a utilização da BIA e a equação proposta por Houtkooper et al. ${ }^{13}$ permitem a estimava da composição corporal com relativa precisão em adolescentes brasileiros com sobrepeso e obesidade.

\section{CONCLUSÃO}

Os resultados do presente estudo mostraram que a BIA utilizando a equação proposta por Houtkooper et al. ${ }^{13}$ foi a que melhor concordou com DEXA e apresentou boa reprodutibilidade para estimar o \%GC, MG e MLG em adolescentes com sobrepeso e obesidade.

\section{AGRADECIMENTOS}

Agradecemos à Fundação Araucária pelo financiamento da pesquisa e ao CNPQ e CAPES pelas bolsas de produtividade à Neiva Leite e à Cláudia Regina Cavaglieri e bolsas de doutorado ao Wendell Arthur Lopes e Larissa Rosa da Silva. Agradecemos ainda ao Centro de Terapias Intensivas (CETI) e ao Colégio da Polícia Militar do Paraná pela disponibilidade do DXA e para o recrutamento da amostra, respectivamente.

Todos os autores declararam não haver qualquer potencial conflito de interesses referente a este artigo.

\section{REFERÊNCIAS}

1. Livingstone B. Epidemiology of childhood obesity in Europe. Eur J Pediatr. 2000;159(Suppl 1):S14-34.

2. Oliveira $C L$, Fisberg M. Obesidade na infância e adolescência: uma verdadeira epidemia. Arq Bras Endrocrinol Metab. 2003;47:107-8.

3. Kiess W, Galler A, Reich A, Müller G, Kapellen T, Deutscher J, et al. Clinical aspects of obesity in childhood and adolescence. Obes Rev. 2001;2(1):29-36.

4. Watts K, Jones TW, Davis EA, Green D. Exercise training in obese children and adolescents: current concepts. Sports Med. 2005;35(5):375-92.

5. Sant'Anna, MSL, Priore SE, Franceschini SCC. Métodos de avaliação da composição corporal em crianças. Rev Paul Pediatr 2009;27(3):315-21.

6. Talma H, Chinapaw MJ, Bakker B, HiraSing RA, Terwee CB, Altenburg TM. Bioelectrical impedance analysis to estimate body composition in children and adolescents: a systematic review and evidence appraisal of validity, responsiveness, reliability and measurement error. Obes Rev. 2013;14(11):895-905.

7. Eisenkölbl J, Kartasurya M, Widhalm K. Underestimation of percentage fat mass measured by bioelectrical impedance analysis compared to dual energy $\mathrm{X}$-ray absorptiometry method in obese children. Eur J Clin Nutr. 2001;55(6):423-9.

8. Lazzer S, Bedogni G, Agosti F, De Col A, Mornati D, Sartorio A. Comparison of dual-energy X-ray absorp- tiometry, air displacement plethysmography and bioelectrical impedance analysis for the assessment of body composition in severely obese Caucasian children and adolescents. Br J Nutr. 2008;100(4):918-24

9. Cleary J, Daniells S, Okely AD, Batterham M, Nicholls J. Predictive validity of four bioelectrical impedance equations in determining percent fat mass in overweight and obese children. J Am Diet Assoc. 2008;108(1):136-9.

10. Schaefer F, Georgi M, Zieger A, Schärer K. Usefulness of bioelectric impedance and skinfold measurements in predicting fat-free mass derived from total body potassium in children. Pediatr Res. 1994;35(5):617-24.

11. de Onis M, Onyango AW, Borghi E, Siyam A, Nishida C, Siekmann J. Development of a WHO growth reference for school-aged children and adolescents. Bull World Health Organ. 2007;85(9):660-7.

12. Tanner JM. Normal growth and techniques of growth assessment. Clin Endocrinol Metab. 1986;15(3):411-51.

13. Houtkooper LB, Going SB, Lohman TG, Roche AF, Van Loan M. Bioelectrical impedance estimation of fatfree body mass in children and youth: a cross-validation study. J Appl Physiol (1985). 1992;72(1):366-73.

14. Deurenberg P, van der Kooy K, Leenen R, Weststrate JA, Seidell JC. Sex and age specific prediction formulas for estimating body composition from bioelectrical impedance: a cross-validation study. Int J Obes. 1991;15(1):17-25 